\title{
Finite Element Modelling and Active Vibration Control of Piezolaminated Plates/Shells
}

\author{
Sudhakar A. Kulkarni and Kamal M. Bajoria \\ Department of Civil Engineering, Indian Institute of Technology Bombay, Mumbai-400076, India
}

(Received 10 June 2002; accepted 30 March 2003)

\begin{abstract}
In this paper, a finite element formulation for shell structures containing integrated distributed piezoelectric sensors and actuators is presented. Higher-order shear deformation theory is used in the formulation, which incorporates the warping of the cross section due to transverse shear stresses, and assumes a parabolic shear strain variation over the thickness. The distributed piezoelectric sensor layer monitors the shape deformation due to the direct effect and the distributed actuator layer suppresses the deflection via the converse piezoelectric effect. The static deflection of the bimorph beam is compared with the literature. The active vibration control performance of the piezolaminated curved beam with distributed sensors and actuators and the variation of the damping effect with different gains and actuator coverage are investigated.
\end{abstract}

\section{INTRODUCTION}

The development of smart composite materials and adaptive structures with sensory/active capabilities has considerable promise to improve the performance and reliability of structural systems. There are great technological implications for this class of structures, which can monitor their own health and take corrective actions. Such smart materials will combine the superior mechanical properties of composite materials with their inherent capability to sense and adapt their static and dynamic response, and continuously monitor the type and location of damage. Some of the presently available smart materials include shape-memory alloys, electrostrictive materials, piezoelectric materials, electro-rheological fluids and magnetostrictive materials.

Various analytical models are available in the literature for analysing the static and dynamic response of smart structures. Analytical models of smart beams with embedded or surface mounted piezoelectric sensors and actuators have been examined in various forms. ${ }^{1-3,7,8}$ Models of plates and shells have been studied using either the classical laminated plate/ shell theory $(\mathrm{CLST})^{\mathbf{2 1}}$ or the first-order shear deformation theory (FOST). ${ }^{\mathbf{1 0 1 6 , 1 8 , 2 1 , 2 8}}$ It is largely established that the higher-order shear deformation theory (HOST) is necessary for predicting through-the-thickness deformation and stress distributions in thick plates more accurately.,12 The HOST improves the in-plane response obtained compared with classical laminated theory even for the case of thin laminated composite plates. $^{15}$

Previous investigations have shown that by using HOST, the tangential in-plane normal stress predictions for various laminated shell problems very closely agrees with elastic solutions. ${ }^{20,23}$ The in-plane displacement distribution through-thethickness using an improved higher-order theory has been studied for laminated composite shells, and the results have indicated improvement over the FOST. ${ }^{22,23}$ The anisotropic and sandwich shells are studied using HOST models, and the accuracy over CLST and FOST is shown in the drastically varying elastic properties from layer to layer., ${ }^{\mathbf{9} 20}$ The finite element analysis of intelligent plate structures using HOST has shown that the method gives better results for both thick and thin piezolaminated composite structures. ${ }^{13}$ HOST finite element models for smart plate structures with eleven mechanical degrees of freedom and one electrical degree of freedom per node are used to study static analysis ${ }^{13}$ and active vibration control. ${ }^{14}$ Studies on piezolaminated beams for shear mode actuators have shown noticeable differences between results from the FOST and HOST models. ${ }^{25}$ The dynamic response of the delaminated smart composite cross-ply beam is studied using the HOST model. ${ }^{26}$ The HOST finite element model developed accounts for the transverse shear through-the-thickness deformations. ${ }^{26}$

To the authors' knowledge, studies of smart shell structures using HOST are not found in the literature. Therefore, this paper presents a finite element model for the analysis of smart shell structures using the HOST model comprising eleven mechanical degrees of freedom per node and one electrical degree of freedom per element. The HOST is based on a Taylor series expansion of generalised displacements and describes the cubic variation of in-plane displacements and the parabolic variation of transverse displacement throughthe-shell thickness. It takes into account warping of the transverse cross sections and leads to the parabolic distribution of transverse shear strains over the thickness of the shell. Thus, shear correction coefficients are not required. A finite-element formulation is presented for modelling the static as well as dynamic effects of a laminated shell structure containing distributed piezoelectric materials subjected to both mechanical and electrical loads. The formulation is derived from the variational principle with the consideration of the total potential energy and the kinetic energy of the structures and the electrical potential energy of the piezoelectric materials. The model is validated for the converse and direct piezoelectric effect using a piezoelectric polymeric PVDF bimorph beam, and the results are compared with the literature. The active vibration control capability of a piezolaminted curved beam is studied and the damping effects for various control gains are demonstrated.

\section{FINITE-ELEMENT FORMULATION}

Figure 1 shows a general smart shell element with composite and piezoelectric layers. The geometry and various co- 